\title{
Numerical Simulation of Temperature Distribution and Material Flow During Friction Stir Welding 2017A Aluminum Alloys
}

\author{
Oussama Mimouni ${ }^{1, a}$, Riad Badji ${ }^{2}$, Mohamed Hadji ${ }^{1}$, Afia Kouadri-David ${ }^{3}$, Hamel Rachid $^{4}$, Nabil Chekroun ${ }^{5}$ \\ ${ }^{1}$ Laboratory of Aircrafts, University of Saad Dahlab, Blida 1, Blida, Algeria. \\ ${ }^{2}$ Center of Industrial Technologies CRTI, P.O Box 64, Cheraga, Algeria \\ ${ }^{3}$ Laboratory of Civil and Mechanical Engineering INSA Rennes, France. \\ ${ }^{4}$ Polytechnic military school Bordj El Bahri Algeria- Algeria. \\ ${ }^{5}$ Research Laboratory of Manufacturing Technology Mechanical / ENPO- Algeria.
}

\begin{abstract}
This study describes the use of fluid dynamic code, FLUENT to model the flow of metal in the AA2017A case around the welding tool pin (FSW). A standard threaded tool profile is used for the analysis of phenomena during welding such as heat generation and flow of the material are included. The main objective is to gain a better understanding of the flow of material around a tool. The model showed a large number of phenomena similar to those of the real process. The model has also generated a sufficient amount of heat, which leads to a good estimate of the junction temperature. These results were obtained using a viscosity which is near the solidus softening.
\end{abstract}

\section{Intoduction}

Friction stir welding (FSW) is recent method of welding in solid state, created and patented by The Welding Institute (TWI) in 1991.

In FSW a cylindrical, shouldered tool with a profiled probe, also called pin, is rotated and slowly plunged into the joint line between two pieces of sheet or plate material, which are butted together. The parts have to be clamped onto a backing bar in a manner that prevents the abutting joint faces from being forced apart. Once the probe has been completely inserted, it is moved with a small tilt angle in the welding direction. The shoulder applies a pressure on the material to constrain the plasticised material around the probe tool. Due to the advancing and rotating effect of the probe and shoulder of the tool along the seam, an advancing side and a retreating side are formed and the softened and heated material flows around the probe to its backside where the material is consolidated to create a high-quality solid-state weld.

\section{2 fluid model}

\subsection{The Arbitrary Lagrangian-Eulerian (ALE) for fluids problems}

Because of its larger capacity, compared to the Eulerian description, of dealing with interfaces between materials and the moving boundaries, the description of the Arbitrary Lagrangian-Eulerian (ALE) is widely used for spatial discretization problems in fluid dynamics and structural. In fact, the method is frequently used in "hydrocodes" to simulate the response of large distortion / deformation of the materials.

The most obvious return of an Arbitrary Lagrangian-Eulerian (ALE) formulation in flow problems is that the convective term must take into account the mesh movement. Also, the grid movement may increase or decrease the effects of convection.

It is important to note that the stress tensor depends on the pressure or on the velocity field according to the viscous or non-viscous flow. This is not the case in solid mechanics. Thus the determination of the stress is not a major concern in the dynamics of Arbitrary Lagrangian-Eulerian (ALE) fluids.

\footnotetext{
a Corresponding author: oussama.mimouni@hotmail.fr
} 


\subsection{Use of the dynamic mesh method with Fluent}

In many works, the simulation and modelling of the FSW welding process are limited through the use of a fixed tool (pin) where the material is modelled as a high viscosity flow of a fluid around the pin. In reality, it's the tool which moves along the welding line. It's this difference which is considered in our simulation $(2 \mathrm{D} / 3 \mathrm{D})$. To deal with this problem of the displacement of the pin in the material, the use of re-meshing method is needed, which allows to gather and re-mesh locally the cells. If the new created cells meet the distortion criterion, the meshing is updated locally with new cells. In this work two methods are used : " local re-meshing " and "local face re-meshing" for 2D /3D simulation.

\subsection{Governing equations}

In order to simplify the numerical simulation process, some supposed conditions are fulfilled:

- The rotational tool is perpendicular to the surface of a weldment;

- The material is considered to be incompressible fluid;

In the FSW process the mass of material is supposed to be a constant, so the equation of mass conservation is as follows:

$\frac{\partial \mathrm{u}}{\partial \mathrm{x}}+\frac{\partial \mathrm{v}}{\partial \mathrm{y}}+\frac{\partial \mathrm{w}}{\partial \mathrm{z}}=0$

where $u, v$ and $w$ mean the velocity of $x$ direction, $y$ direction and $z$ direction, respectively.

The momentum conservation equation can be described with the Navier-Stokes equation. The expression of the equation is as follows:

$$
\left\{\begin{array}{c}
\frac{\partial u}{\partial t}+u \frac{\partial u}{\partial x} v+\frac{\partial u}{\partial y}+w \frac{\partial u}{\partial z}=F_{x}-\frac{1}{\rho} \frac{\partial p}{\partial x}+\mu\left(\frac{\partial^{2} u}{\partial x^{2}}+\frac{\partial^{2} u}{\partial y^{2}}+\frac{\partial^{2} u}{\partial z^{2}}\right) \\
\frac{\partial v}{\partial t}+u \frac{\partial v}{\partial x} v+\frac{\partial v}{\partial y}+w \frac{\partial v}{\partial z}=F_{y}-\frac{1}{\rho} \frac{\partial p}{\partial y}+\mu\left(\frac{\partial^{2} v}{\partial x^{2}}+\frac{\partial^{2} v}{\partial y^{2}}+\frac{\partial^{2} v}{\partial z^{2}}\right) \\
\frac{\partial w}{\partial t}+u \frac{\partial w}{\partial x} v+\frac{\partial w}{\partial y}+w \frac{\partial w}{\partial z}=F_{x}-\frac{1}{\rho} \frac{\partial p}{\partial z}+\mu\left(\frac{\partial^{2} w}{\partial x^{2}}+\frac{\partial^{2} w}{\partial y^{2}}+\frac{\partial^{2} w}{\partial z^{2}}\right)
\end{array}\right.
$$

The energy conservation equation can be expressed as follows:

$$
\rho c\left(\frac{\partial T}{\partial t}+u \frac{\partial T}{\partial x} v+\frac{\partial T}{\partial y}+w \frac{\partial T}{\partial z}\right)=\lambda\left(\frac{\partial^{2} T}{\partial x^{2}}+\frac{\partial^{2} T}{\partial y^{2}}+\frac{\partial^{2} T}{\partial z^{2}}\right)
$$

where $c$ is the specific heat of material, $\lambda$ is the thermal conductivity of material and $T$ is the temperature of fluid.

In this work, a 2D/3D model of welding FSW of aluminum alloy AA2017A was developed and validated by a transient simulation with fully coupled materials flow and taking into account the heat flow generated by friction. The material is assumed to be viscoelastic, of which the viscosity was obtained from tests on a dynamic mechanical analyzer (DMA). By combining the two formulations Euler and Lagrange, a dynamic mesh method (see figures 1 and 2) was used to model the flow of material induced by the movement of the threaded pin. The use of transient model allows not only displaying the complex movement of threaded pin, but also the simulation of heat and material flow during welding. This approach aims getting better prediction and representation of thermomechanical history of the material, which is necessary in the microstructure and final texture analysis. The equations governing the $2 \mathrm{D} / 3 \mathrm{D}$ transient heat transfer and material flows are discretized using the finite volume method and solved using fluid dynamics solver «ANSYS / FLUENT ».

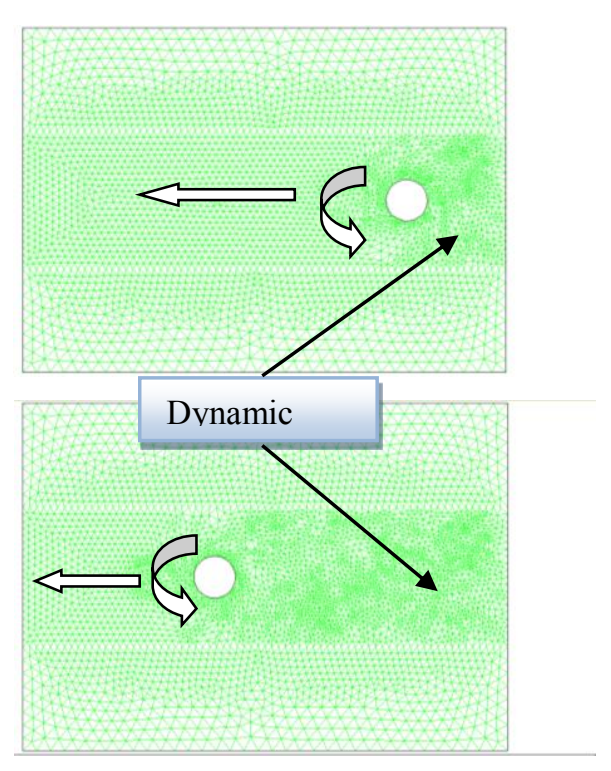

Figure 1.Evolution of 2D dynamic mesh. where $F_{x}, F_{y}$ and $F_{z}$ are the volume force of $x$ direction, $y$ direction and $z$ direction, respectively, $p$ is the static pressure of the flow field, $\mu$ is the viscosity of fluid, $\rho$ means the mass of material. 

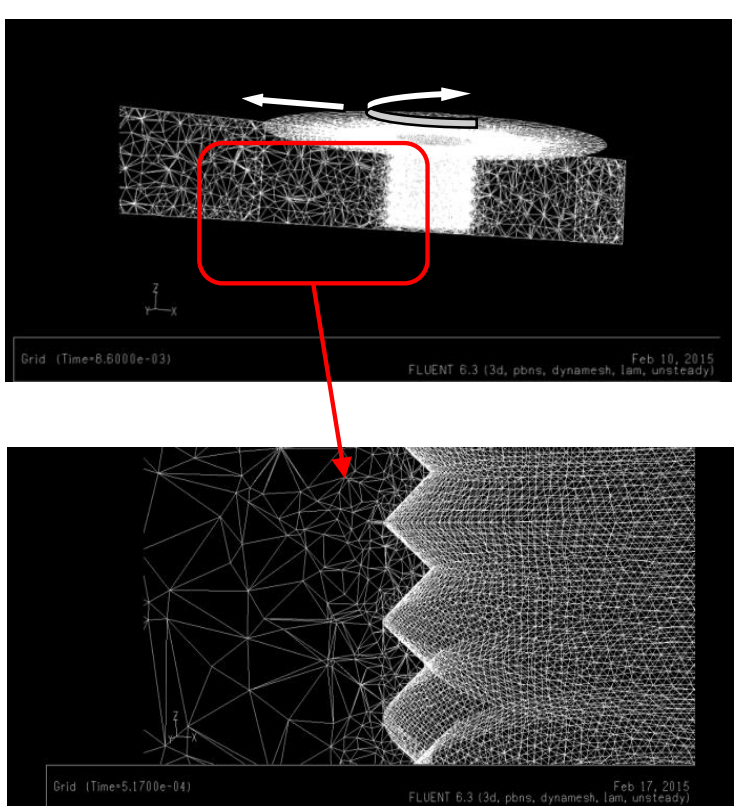

Figure 2.Evolution of 3D dynamic mesh.

\subsection{Heat generation model}

In the present model, a heat flux is applied on the outer surfaces of the pin and the shoulder which are in contact with the material.

In the FSW process, the heat is generated near the contact surfaces, which may have complex geometries depending on the tool shape. However, for the used model, the surface of the tool are assumed to be cylindrical with a horizontal shoulder. The conical shoulder surface is characterized by the angle $\alpha$, which is set to zero in the case of a flat shoulder.

The simplified tool is shown in Figure 3, where $R_{\text {shoulder }}$ is the radius of the shoulder, and $R_{P I N} H_{P I N}$ and are respectively the radius and height of the pin. In this way total heat output can be calculated as follows :

$$
Q_{\text {total }}=2 / \pi \omega \tau_{\text {contact }}\left(R_{\text {shoulder+ }}^{3} 3 R_{\text {probe }}^{3} H_{\text {probe }}\right)
$$

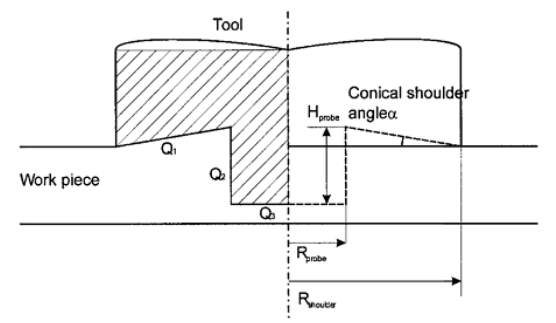

Figure 3. Heat flow represented in a simplified FSW tool.[3]
The numerical modeling of FSW flow can be based on models and technics used for other processes such as friction welding, extrusion, machining, forging, rolling and ballistic impact [2]. As for the analysis of heat flow, numerical flow models can use for meshing either the Eulerian formulation, Lagrangian formulation, or a combination of both (hybrid solution Lagrange-Eulerian).

Through the use of fluid dynamic analysis models (CFD) instead of solid mechanical models, some mechanical effects are excluded from the analysis, such the forging effect. In addition, these models cannot predict the needed force because the elasticity is neglected.

One of the difficulties of the numerical analysis is the strong gradient of the flow velocity near the tool. To solve this problem, the mesh areas is divided in several regions, as shown in Figure 4. The flow around the tool is a predominantly rotational, the meshing is adapted to the tool displacement. This rotation region is made large enough to contain the entire deformation zone where the mesh size is much finer [4].

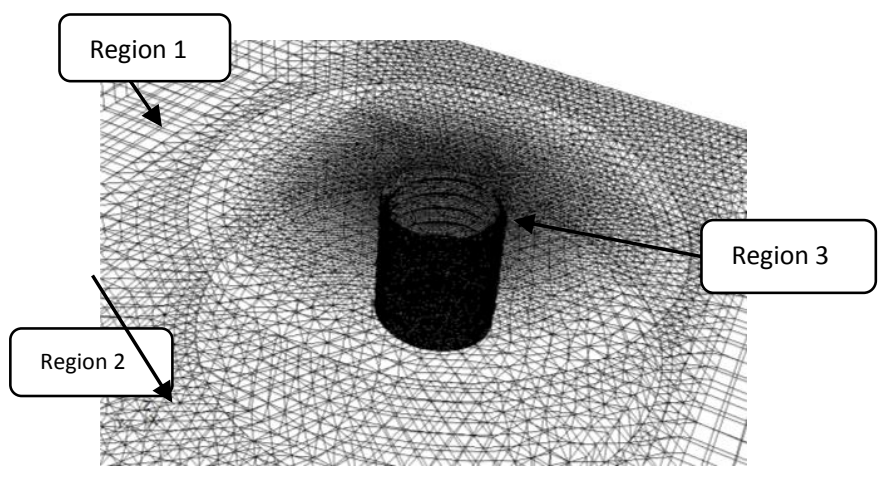

Figure 4. Discretization of the plate into differents zones.

A 3D analysis using the finite volume method based on an Arbitrary Lagrangian-Eulerian (ALE) formulation, provided results with an interesting physical insight. Also, the 3-D analysis is able to handle some of the process complexities such as a concave shoulder, tool tilt, and different threaded pin profiles.

A numerical simulation of the 3-D FSW method, presented by Riahi and Nazari [5], relates to studying the impact of tool speed, the heat flux and the residual stress distribution. Another interesting study shows a 3-D thermomechanical model FSW based on CFD analysis [6]. This model describes the material flow around the tool during the welding operation. An alloy of AA2024 aluminum plate of $3.2 \mathrm{~mm}$ thick is used. The minimum and maximum values of the workpiece temperature (near the tool shoulder) are shown in Figure 5, 
where one can see that the maximum temperature value decreases as the welding speed increases. On the other hand, the maximum value of the temperature increases when the tool rotation speed increases.
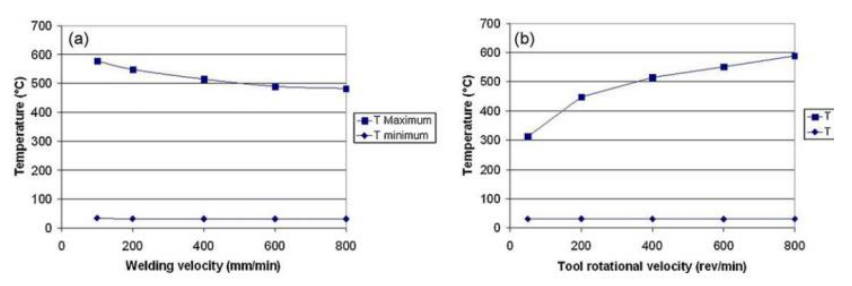

Figure 5. Extreme temperatures in the welds; (a) as a function of the welding velocity of the tool for a tool rotational velocity equal to $400 \mathrm{rpm}$; (b) as a function of the tool rotational velocity for a welding velocity equal to $400 \mathrm{~mm} / \mathrm{min}$. [6]

The modeling of the FSW welding process allows to visualize the fundamental behavior of the welded materials, and to study the influence of different welding parameters, including the design of the tool and boundary conditions, without performing costly experiments. The FSW modeling is a difficult task because of its multiphysics features. The process combines heat flow, high temperature, plastic deformation and microstructure evolution. Nowadays, the numerical simulation of the FSW process may not only be used to optimize the process. Increased knowledge produced based on the FSW process simulation can lead in the near future, to replace experimental tests. This will help promoting and expanding the FSW process to a wider range of different applications.

\section{Geometry and boundary conditions}

The purpose is to perform the simulation using 2D and $3 \mathrm{D}$ modeling with FLUENT software, and to present the different results obtained.

The sheet is modeled as a rectangle of length $\mathrm{L}=70 \mathrm{~mm}$ and $\mathrm{b}=50$ width and thickness $\mathrm{t}=6 \mathrm{~mm}$. A ring of outer diameter $\mathrm{D}=22 \mathrm{~mm}$ is drawn on the upper surface of the sheet, which corresponds to the shoulder. The figure 6 shows the surfaces designation that will be used thereafter. The pin is modeled as a cylinder of diameter $\mathrm{d}=6 \mathrm{~mm}$ and height $\mathrm{h}=5.7 \mathrm{~mm}$.

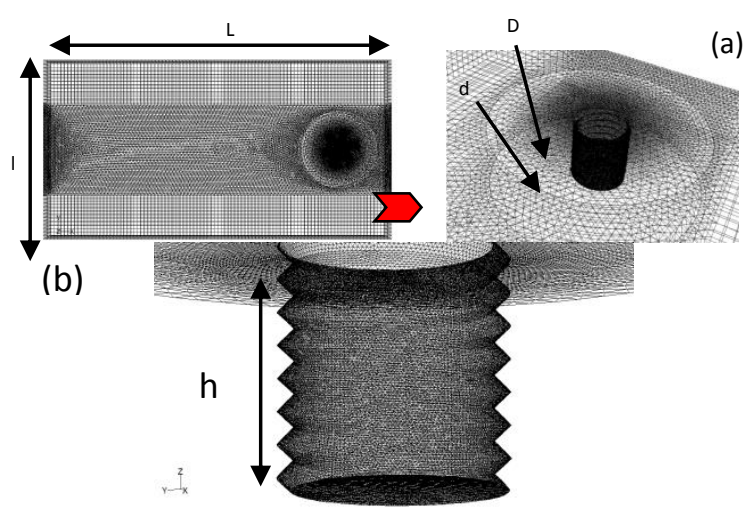

Figure. 6 Perspective of model mesh (a) and details of pin meshed zones (b) Thread tool.

In FLUENT 3D model, the origin is in the center of the sheet and at mid-thickness. Thus, knowing the rotation direction of the tool and its advancing speed, the AS-side and the RS-side can be differentiated by positive or negative $\mathrm{Y}$ values.

The meshing of reference used in the calculations is shown in figure 7. It consists of 394427 cells (tetrahedral / square) with minimum edge size of $0.1 \mathrm{~mm}$.

A dynamic mesh was used to capture the two movements of the axis of the tool rotation and translation. Thus, the mesh is modified continuously over the time as shown in Figure 7. The dynamic mesh is composed of two regions inner and outer regions. The inner region contains the contour of the tool pin (engaged with tetrahedral elements) and the outer region contains the tool shoulder. The use of such a dynamic mesh combines the advantages of the two formulations of Lagrange and Euler. In other words, the movement of the tool is correctly taken into account with the movement of Lagrangian elements, while at the same time the formulation of Euler allows the material to flow through the mesh, by maintaining good mesh quality.

The transient model was used to simulate the movement of the tool. Heat flow, defined in equation (4) was applied to the tool surfaces. For the upper and side surfaces of the plate, the thermal boundary condition was fixed by convection between the air and the plate, with a heat transfer coefficient of $30 \mathrm{~W} / \mathrm{m}^{2}{ }^{\circ} \mathrm{C}$.

For the lower surface of the plate, the heat transfer coefficient by convection between the plate and the anvil plate was set at $150 \mathrm{~W} / \mathrm{m}^{2}{ }^{\circ} \mathrm{C}$. The value of the heat transfer coefficient is found to be similar to 
that reported in the literature for modeling FSW aluminum alloy 2017A. The plate was initially at room temperature of $27^{\circ} \mathrm{C}$.

In the simulation process using the FLUENT software, the approach is to consider the metal material as a fluid, which is under the effect of the rotation and advance of the tool. The fluid is stationary and the tool has an advancing speed of $0.6 \mathrm{~mm} / \mathrm{s}$ and a rotational speed of $1250 \mathrm{tr} / \mathrm{min}$.

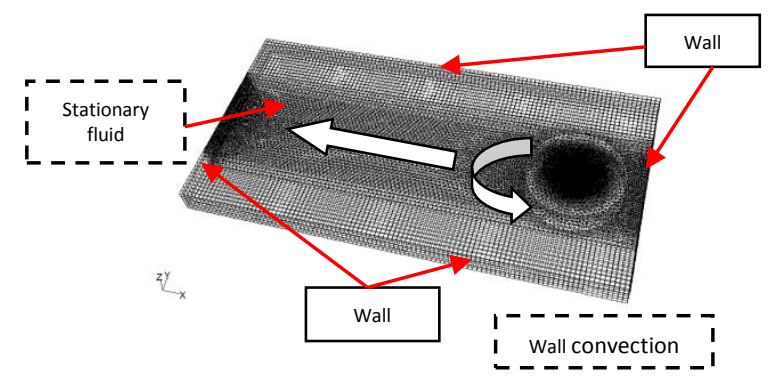

Figure7. Mesh generation of model used in simulation.

\section{Material parameters}

For the model based on the finite volume Euler formulation, the main parameters are the specific heat material, the thermal conductivity and the viscosity coefficient as a function of the temperature. The latter is derived from the experiment performed on the DMA machine (see figure 8), that allows identifying and quantifying the dynamic viscosity of the material depending on the temperature, which is used directly into the numerical model.

\subsection{Dynamic mechanical analysis}

The DMA is used to determine the evolution of the viscoelastic properties as a function of temperature, frequency or time, by applying cyclic deformation [1]. The viscoelastic behaviour is generally described as a complex modulus $E^{*}=E^{\prime}+i E^{\prime \prime}$. The real part $E^{\prime}$ called storage modulus, reflects the conservative elastic behaviour of the material. As for the imaginary part $\mathrm{E}^{\prime \prime}$ called loss modulus, characterizes the viscous and dissipative behaviour. The quotient $E^{\prime \prime} / E^{\prime}=\tan \delta$ is called loss factor.

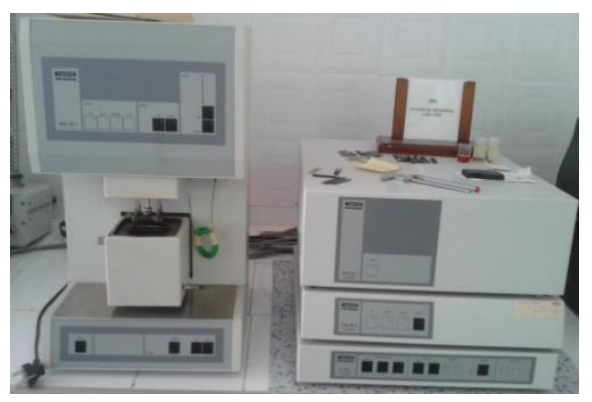

Figure 8. Dynamic Mechanical Analyzer (DMA).

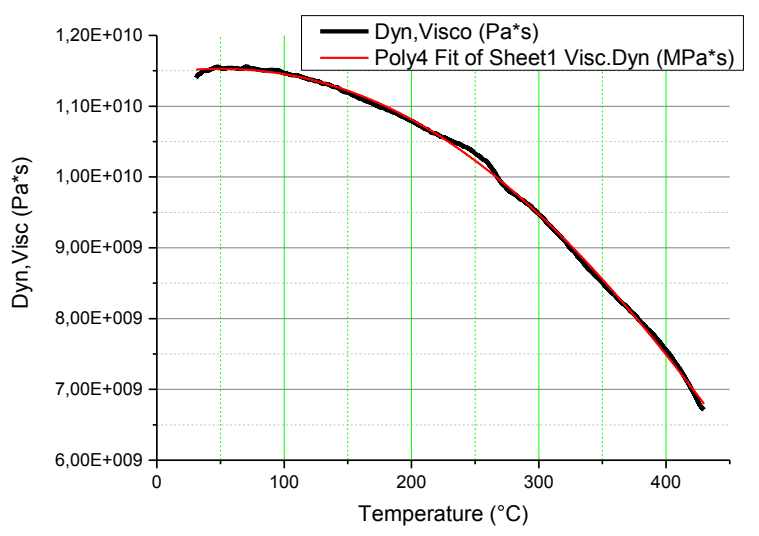

Figure 9. Dynamic viscosity if AA2017A obtained from DMA test.

The viscosity in term of the temperature can be expressed in polynomial form as :

$\mu($ Pa.s $)=1,1463210^{+10}+2,488410^{+6} T-22117,33216 T^{2}$

$-43,29134 \mathrm{x} T^{3}+0,05236 T^{4}$

The density is $2800 \mathrm{~kg} / \mathrm{m}^{3}$. The specific heat $\left(C_{p}\right)$ and the thermal conductivity $(\lambda)$ at different temperatures are calculated using equations. (6) and (7) [7].

$\lambda=103.264+0.241 T$

$C_{p}=754.08+0.3729 T+0.0012 T^{2}$

\section{Results and discussion}

As shown in figure 10, the solution reaches the stationary state when the mechanical equilibrium is attained after a number of additional iterations. It also shows the evolution of minimum, maximum and average temperatures calculated on the entire field of study. 


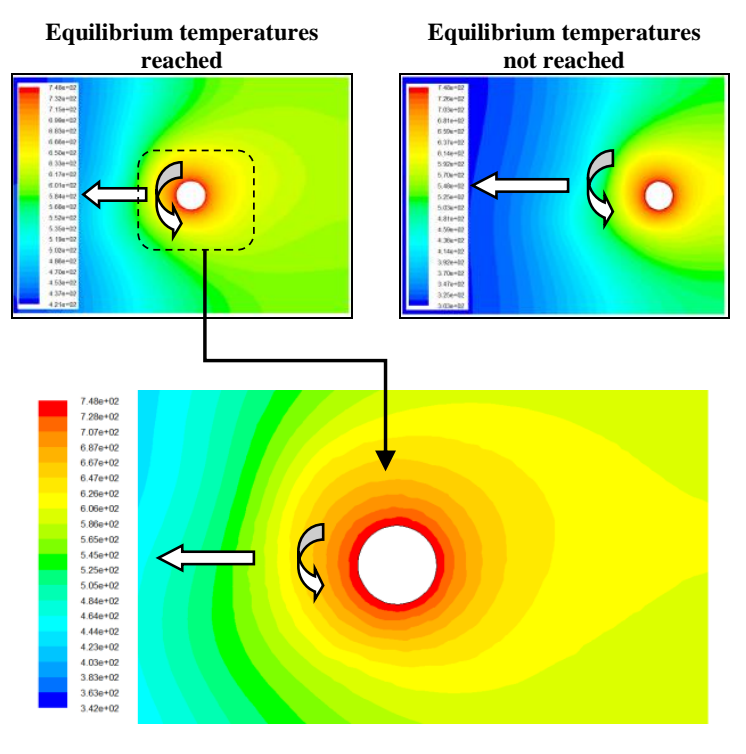

Figure 10. Temperature distribution during FSW welding.

The comparison between the predicted temperatures with the experimental data is presented in Figure 12. The temperature values are measured on the upper surface at the points $\mathrm{T} 1$ and $\mathrm{T} 2$ of the plate, as represented in Figure 11. One can observe a reasonable agreement between the temperature values obtained with the model with those measured in experiment. The heat generated during FSW must be sufficiently enough to enable softening, stirring and mixing the material.

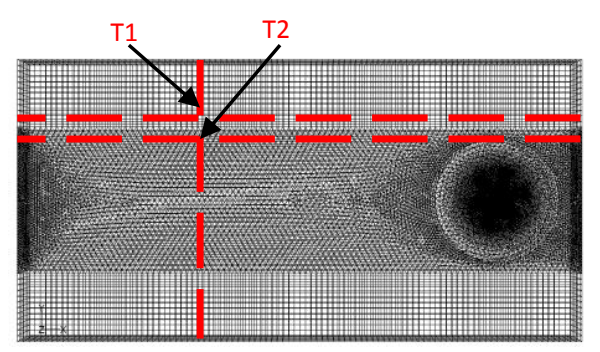

Figure 11. Thermocouple's positions along plate.

The calculated temperature profiles along the upper surface sections of the cross-piece through the tool axis are shown in Figure 12.
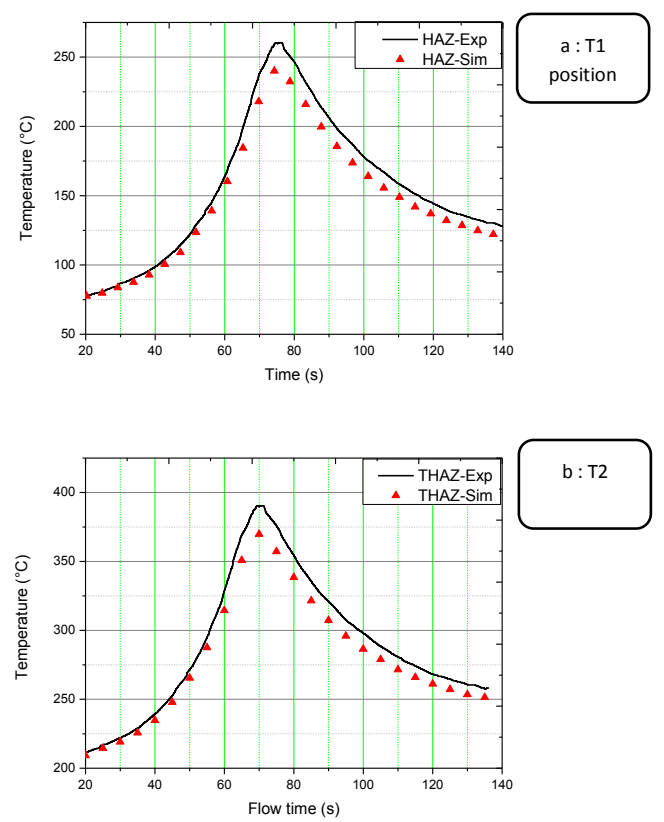

Figure 12. Comparison between the measured and predicted temperatures: (a) HAZ zone, (b) THAZ zone.

The maximum temperature is located in the area where the deformation is greater. A temperature peak of $475^{\circ} \mathrm{C}$ is recorded in the area close to the tool (shoulder, pin). The temperature decreases when going far from the mixing zone. The temperature distribution is asymmetric, which is a characteristic of the welding process.

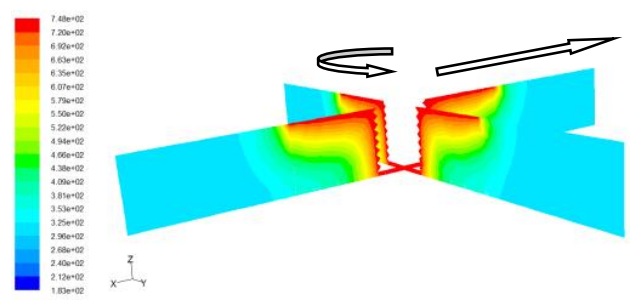

Figure 13.Cross-section along the axis $(\mathrm{X})$ and $(\mathrm{Y})$.

The contours of the velocity vectors through a cross-sectional below the tool shoulder are shown in Figures 14 and 15 respectively for 2D / 3D cases. This analysis is only intended to be illustrative. The slipping was simulated by prescribing the speed to the shoulder and to the tool pin. These figures illustrate several flow characteristics. First, the greater speed of rotation is always in the region of the greater deformation, namely under the shoulder and around the pin. Also, one can distinguish a phenomenon rarely shown in the literature, namely the creation of vortices between each pin thread pitch, as seen in Figure 16. 


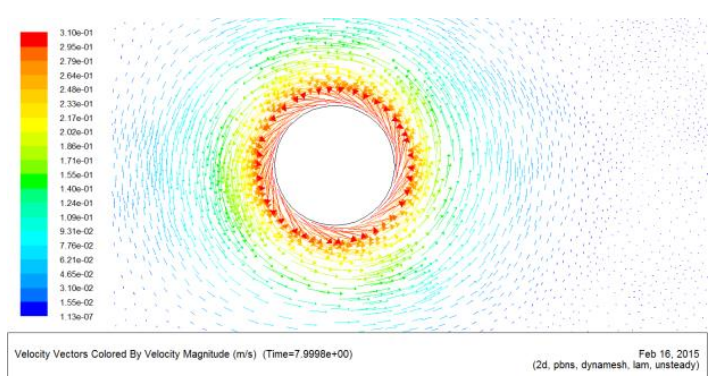

Figure 14. Velocity vectors for the $2 \mathrm{D}$ case.

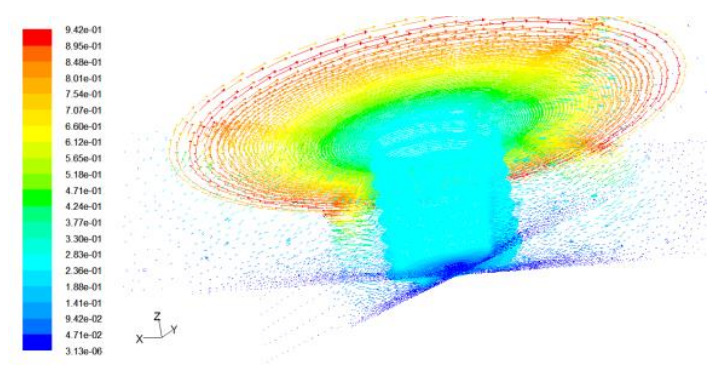

Figure 15. Velocity vectors for the 3D case.

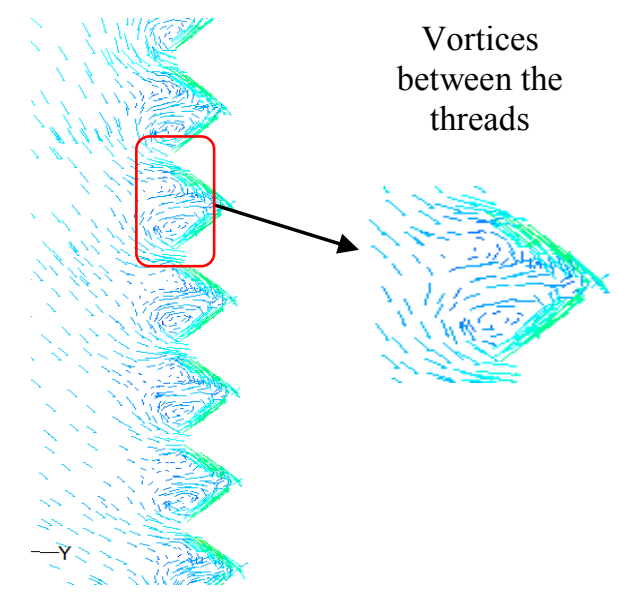

Figure 16. Vortices apparition between threads.

The figure 17 shows an instantaneous material flow caused by the displacement of FSW welding tool. The 3D visualization shows precisely the material flow around the tool and through the pin threads.

This figure also shows the streamlines around the tool, which illustrate the path of the material particles passing through the plate stirring region. The upward effect of the material flow produced by the pin thread is well illustrated too. We can observe that once the flow reaches the upper surface in contact with the tool shoulder, it takes a descending path and turns several times before continuing its course. Accordingly, the material remains near the surface of the tool shoulder. This kind of flow patterns was observed by Guerra et al [8]. It has been demonstrated the creation of a rotational area around the shoulder, where the material undergoes several turns before deposited in the pin wake. In addition, one can observe how the flow in the lower half of the plate is directed through the interaction with the pin threads, while in the upper part is driven by the interaction with the tool shoulder. Thus, it is possible to visualize the flow patterns without using tracing particles.
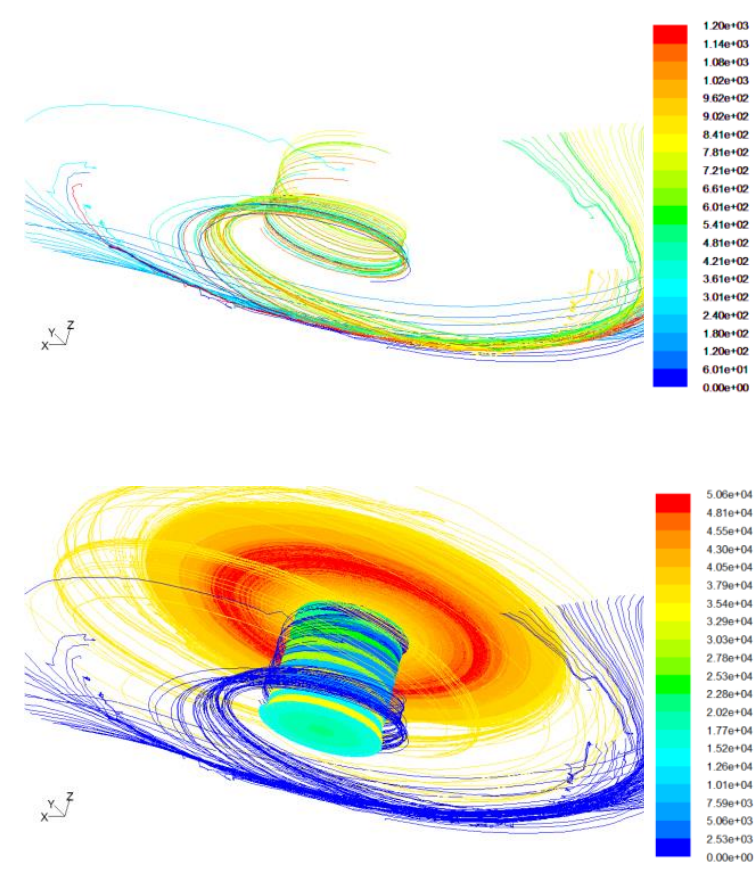

Figure 17.Streamlines of the material flow.

\section{Conclusion}

A 2D/3D simulation for FSW process model based on fluid mechanics has been presented in this paper. A friction stir tool with threaded tapered pin, similar to the real friction stir tool, was used in the mathematical modeling to explore the general material flow rule. The method of dividing the welding zone in several regions combining the velocity field and viscosity field is used in this paper.

A numerical procedure for the visualization of material flow during the FSW process is presented. The technique based on particle streamline tracing is applied to track particle trajectories, at the post process level once the velocity field is obtained.

The proposed method is validated through comparing the results of a $2 \mathrm{D}$ FSW modeling to experimental data. The $2 \mathrm{D}$ simulation shows that the material flow is not symmetric about the welding line. The flow patterns in the advancing and retreating sides are different. The welding join is created straightly at the center line and no material mixing is observed. These results are found to be in agreement with experimental data. 
The particle streamline tracing method is also applied to a $3 \mathrm{D}$ simulation including the complete tool geometry and threaded pin. This simulation has shown some distinctive 3D features of the flow. The material flow around the pin has a centrifugal shape across the thickness. The material stirred on the advancing side performs more than one revolution around the tool. The up and down of the material movement in the thickness direction is also observed.

In both $2 \mathrm{D}$ and $3 \mathrm{D}$ simulations, agreement with experimental evidence is remarkably good, showing that particle tracing is a useful tool giving good insight of the FSW process.

\section{References}

1. K.P. Menard: CRC PRESS Boca,Library of Congress Cataloging-in-Publication Data, USA, (1999)

2. Mishra RS, Ma ZY (2005) Friction stir welding and processing. Mater Sci Eng R 50:1-78. doi: 10.1016/j.mser.2005.07.001

3. Assidi M, Fourment L (2009) Accurate 3D friction stir welding simulation tool based on friction model calibration. Int J Mater Form 2:327-330. doi: 10.1007/s12289-009-0541-6

4. Colegrove PA, Shercliff HR (2005) 3Dimensional CFD modelling of flow round a threaded friction stir welding tool profile. J Mater Proces s Tech 169(2):320-327. doi: 10.1016/j.jmatprotec.2005.03.015

5. Riahi M, Nazari H (2011) Analysis of transient temperature and residual thermal stresses in friction stir welding of aluminum alloy 6061T6 via numerical simulation. Int J Adv Manuf Technol 55:143-152. doi: 10.1007/s00170010-3038-z

6. Jacquin D, de Meester B, Simar A, Deloison D, Montheillet F, Desrayaud C (2011) A simple Eulerian thermomechanical modeling of friction stir welding. J Mater Process

Tech 211(1):57-65. doi: 10.1016/j.jmatprotec.2010.08.016

7. S.d.ji, and $\mathrm{Al}$, "numerical simulation of material flow behaviour of friction stir welding influenced by rotational tool geometry", computational materials science 63 218-226, 2012
8. Guerra, M. et al, Flow patterns during friction stir welding, Materials Characterizations, v.49, p.95-101, 2003. 